\title{
Optimization of Naked DNA Delivery for Interferon Subtype Immunotherapy in Cytomegalovirus Infection
}

\author{
Emmalene J. Bartlett ${ }^{1}$, Vanessa S. Cull ${ }^{1}$, Eva N. Mowe ${ }^{1}$, Josephine P. Mansfield ${ }^{1}$ and \\ Cassandra M. James ${ }^{1 *}$
}

${ }^{1}$ Division of Veterinary and Biomedical Sciences, Western Australian Biomedical Research Institute, Murdoch University, Perth, Western Australia, Australia

*To whom correspondence should be addressed: Dr C. James, Division of Veterinary and Biomedical Sciences, Murdoch University, South St, Murdoch 6150, Western Australia, Australia. Tel: 618- 9360 2267; Fax: 618- 9310 4144; Email: casjames@central.murdoch.edu.au

Submitted: December 10, 2002; Revised: February 3, 2003; Accepted: February 5, 2003; Published: February 17, 2003

Indexing terms: interferons, gene therapy, cytomegalovirus, DNA.

\begin{abstract}
Type I interferon (IFN) gene therapy modulates the immune response leading to inflammatory heart disease following cytomegalovirus (CMV) infection in a murine model of postviral myocarditis. Efficacy of different immunisation protocols for the IFN constructs was influenced by the dose of DNA, subtype choice, combination use, pre-medication, and timing of DNA administration. Optimal efficacy was found with bupivacaine treatment prior to DNA inoculation of $200 \mu \mathrm{g} I F N$ DNA 14 days prior to virus challenge. Maximal antiviral and antimyocarditic effects were achieved with this vaccination schedule. Furthermore, inoculation of synergistic IFN subtypes demonstrated enhanced efficacy when delivered either alone or with CMV $g B$ DNA vaccination in the CMV model. Thus naked DNA delivery of IFN provides an avenue of immunotherapy for regulating herpesvirus-induced diseases.
\end{abstract}

\section{INTRODUCTION}

Cytomegalovirus (CMV) is a herpesvirus that causes latent and persistent infection. This virus may induce inflammation of the heart known as myocarditis, which may lead to dilated cardiomyopathy, arrhythmias and sudden death. Currently, there is no effective safe vaccine for CMV, as the vaccines studied so far, only provide partial protective immunity upon challenge with infectious virus. Vaccines for viral infections and diseases have encompassed live attenuated viruses, subunit virions, recombinant viral proteins/peptides and recently viral
DNA constructs. Interest in cytokines has increased with the aim of seeking improvement of the efficacy of such viral DNA vaccines. Nonetheless, optimization of DNA delivery is an important aspect of vaccine development. Specifically in the case of DNA vaccines, factors including DNA dosage, choice of cytokine genes, pre-medication, and timing of DNA administration can influence the immune response following intramuscular (i.m.) vaccination.

Many studies of murine models have found that effective DNA vaccination occurs in a dose-dependent manner, generally with a dose range of 25-200 $\mu$ g plasmid DNA (1-4). Previously, we have found in vivo expression of interferon alpha (IFNA) genes following i.m. inoculation of $200 \mu \mathrm{g}$ naked DNA to be effective against murine cytomegalovirus (MCMV) infection. This approach also allows us to examine the superiority of individual and combinational usage of different IFN subtypes within the multigene family (5-9). Other groups using type I IFN-expressing plasmids have reported effective inoculation with $100 \mu \mathrm{g}$ i.m. (10) or $100 \mu \mathrm{g} /$ eye (11) in cancer and herpes simplex virus (HSV) infection, respectively. In addition, investigators have found a dose-response effect of pCMVIFN $\alpha 1$ plasmid on HSV-1 replication in the range of 25 $100 \mu \mathrm{g} /$ eye (11).

The choice of cytokines is very important in providing protective immunity via immunotherapy. Since type I IFNs belong to a multigene family, we examined the effect of seven individual IFN subtypes (IFNA1, A2, A4, A5, A6, A9 and B), administered by DNA inoculation, on systemic MCMV infection and myocarditis (8). We found that IFNA6 treatment

(C) 2003. Biological Procedures Online. Published in Biological Procedures Online under license from the author(s). Copying, printing, redistribution and storage permitted.

Biological Procedures Online • Vol. 5 No. $1 \bullet$ February 17, 2003•www.biologicalprocedures.com 
reduced MCMV replication whilst IFNA5 and $A 2$ treatment enhanced virus replication. Moreover, IFNA6, $A 9$, and $B$ treatment inhibited acute myocarditis whilst IFNA6 was able to also reduce chronic cardiac inflammation. In addition, viral DNA vaccines encoding the CMV glycoprotein B (gB) are currently being investigated and require improved efficacy. We have previously studied the efficacy of the $g B$ DNA vaccine with the application of type I IFNs, IFNA6, IFNA9 and IFNB (9). CMV-induced myocarditis was preferentially reduced with either IFNA9 or IFNB but not with IFNA6, coimmunisation. However, IFNA6, IFNA9 and IFNB markedly reduced chronic myocarditis in CMV $g B$-vaccinated mice.

Pre-medication with bupivacaine prior to DNA inoculation is also a factor in the optimization of the DNA inoculation protocol. Bupivacaine is an anaesthetic which destroys myofibres, induces myogenesis and is reported to enhance protein expression following DNA inoculation since regenerating muscle is thought to encourage DNA uptake (12). Reports of the effect of bupivacaine pre-medication on transgene expression demonstrate conflicting evidence with some reports suggesting no effect and some observing up to 40 -fold increase in gene expression $(6,12)$.

Previous reports have demonstrated that the timing of DNA inoculation contributes to therapeutic outcome. In particular, timing of DNA inoculation can influence the type of Th cell response induced. This was first demonstrated with plasmid encoded GM-CSF (pGM-CSF) co-administered with DNA encoding the envelope protein of HIV. Inoculation of pGMCSF 3 days prior to DNA vaccination induced a predominantly Th2-type immune response, simultaneous administration with antigen induced both a Th1 and Th2 type response and inoculation 3 days after DNA vaccination stimulated a predominantly Th1 response (13). A similar effect has since been observed with plasmid administered IL-12 in models of HIV and Leishmania vaccination $(1,2)$. Similarly, previous studies in our laboratory have demonstrated variability in the immunomodulatory efficacy of type I IFN subtype transgenes, which is dependent on the timing of DNA inoculation in relation to MCMV challenge (5).

In this study, we determined the optimal inoculation schedule for type I IFN subtype DNA constructs in the MCMV model of infection and disease. We found that DNA dosage, bupivacaine pre-medication, and timing of DNA inoculation (5), were contributing factors in the therapeutic efficacy of IFN DNA therapy in regulating herpesvirus infection and disease sequelae. Furthermore, we examined the synergistic effects of $g B, I F N A 6$ and $I F N B$ compared to that of the $g B$ DNA vaccine alone. We found that the combined $g B / I F N A 6 / I F N B$ DNA vaccine was superior to the $g B$ DNA vaccine in providing protection from MCMV infection. Further applications of the synergistic properties provided by type I IFNs may be used in conjunction with other viral DNA vaccines for improved vaccine efficacy.

\section{MATERIALS AND METHODS}

\section{Mice}

Specific pathogen-free inbred male BALB/c mice of 4 weeks of age were supplied by the Animal Resources Centre (Murdoch, Western Australia).

\section{Virus}

The K181 strain of MCMV was prepared as a salivary gland homogenate by passage through 3-week-old female BALB/c mice and stored under liquid nitrogen. MCMV titres in target organs were quantitated by plaque assay using mouse M210B4 stromal cells. Virus titres are expressed as mean pfu/g tissue \pm SEM ( 5 mice per group). The limit of detection was $100 \mathrm{pfu} / \mathrm{g}$ for liver and spleen and $6400 \mathrm{pfu} / \mathrm{g}$ for salivary gland tissue.

\section{Expression plasmid DNA constructs}

IFN subtype genes and the full-length gB MCMV gene were cloned into the mammalian expression vector, pkCMVint (VICAL, San Diego, CA) as previously described $(8,9)$. Large-scale plasmid preparations were obtained from terrific broth cultures of transformed E.coli (DH-5 $\alpha$ ) using standard DNA extraction procedures with $\mathrm{LiCl}$ precipitation. DNA integrity was checked by agarose gel electrophoresis and concentrations determined by spectrophotometric analysis.

\section{DNA immunisation and virus challenge protocol in vivo}

To induce muscle regeneration, mice were injected bilaterally with $20 \mu \mathrm{L} 0.5 \%$ bupivacaine 5 days prior to inoculation of DNA constructs (either 50, 100, 200 or $400 \mu \mathrm{g} / \mathrm{mouse}$ as indicated). DNA was diluted in a $50 \mu \mathrm{L}$ volume of pyrogen-free saline and injected bilaterally in tibialis anterior (TA) muscles. Mice were either treated with the Blank expression vector or vectors expressing IFN subtypes (IFNA6, $A 9$ and $B$ ) or the $g B$ transgene alone or in combination. At 14 days post DNAtreatment, mice were inoculated with $100 \mu \mathrm{L}$ of $1 \times 10^{4} \mathrm{pfu}$ MCMV, diluted in pyrogen-free saline, by the intraperitoneal (i.p.) route (5 mice/group). At days 3, 7, 10 and 56 postinfection (p.i.), tissues were collected. Deviations from this standard protocol are stated in the results and figure legends.

\section{Myocarditis analysis}

Hearts from infected mice were removed at day 7 and 56 p.i., transected along the midline and fixed overnight in Bouin's fluid, transferred to $70 \%$ ethanol and then processed into paraffin blocks. Sections were stained with haematoxylin and eosin (H\&E) and evaluated for evidence of cellular inflammation and necrosis as previously described (8). Two heart sections were scored for each animal. Myocarditis was 
evaluated histologically as the mean number of inflammatory foci per heart section \pm SEM (5 mice/group).

\section{Statistical analysis}

Levels of significance $(\mathrm{p}<0.05)$ were determined by the unpaired student $t$-test assuming unequal variance between the means.

\section{RESULTS AND DISCUSSION}

\section{Results}

\section{Importance of IFN dose for efficacy of DNA delivery in vivo}

Previously, we have demonstrated efficacy using $200 \mu \mathrm{g} I F N$ transgenes inoculated i.m. Here, we evaluated IFN transgene inoculation using lower doses of DNA for therapeutic efficacy in reducing virus titres and cardiac inflammatory foci. Mice were treated with either $50 \mu \mathrm{g}$ or $100 \mu \mathrm{g}$ Blank, IFNA6 or IFNB DNA and challenged with a sublethal dose of MCMV ( $1 \times 10^{4}$ pfu i.p.). The effect of low doses of IFNA6 and IFNB on early MCMV replication and acute-phase myocarditis was evaluated in comparison to the Blank vector. Acute-phase viral titres were determined by plaque assay of spleen and liver homogenates at day 3 p.i. and salivary gland homogenates at day 7 p.i. and acute phase myocarditis was evaluated at day 7 p.i.

The antiviral efficacy previously demonstrated by these IFN subtypes at $200 \mu \mathrm{g}$ per mouse (8) was suppressed at these lower doses. More specifically, IFNA6 and IFNB DNA had no significant effect on viral titres in any tissue examined (Fig. $1 \mathrm{~A}, \mathrm{~B}$ and C). IFNA6 at $50 \mu \mathrm{g}$ reduced liver titres by only 1.6fold and at $100 \mu \mathrm{g}$ marginally reduced both spleen (2.0-fold) and liver (1.3-fold) titres compared to Blank-vector treated mice. Whereas, treatment with IFNA6 at $200 \mu \mathrm{g}$ was most effective with significant reductions of 5.7- and 19-fold of virus titres in the spleen and liver, respectively. However, treatment with $I F N B$ at either $50 \mu \mathrm{g}, 100 \mu \mathrm{g}$ or $200 \mu \mathrm{g}$ did not affect virus titres in any tissue tested. Administration of $200 \mu \mathrm{g}$ of either IFNA6 or IFNB has been previously associated with suppression of acute stage myocarditis with 4.7- and 2.4-fold reduction, repectively compared to Blank vector-treated mice (8). Here, we show that myocarditis was also significantly reduced with inoculation of $50 \mu \mathrm{g} /$ mouse of either IFNA6 (4.3fold) or IFNB (1.7-fold) DNA in comparison to Blank vectortreated mice. However, treatment with $100 \mu \mathrm{g} I F N$ was not effective with only a 1.4-fold reduction in comparison to control groups (Fig. 1D). Similar to our previous findings, viral titre was not an indicator of disease. In brief, reducing the dose of IFN DNA to $50 \mu \mathrm{g} /$ mouse or $100 \mu \mathrm{g} /$ mouse reduced the antiviral and antimyocarditic efficacy of IFNA6 and IFNB that we have previously observed with the higher dose of $200 \mu \mathrm{g} /$ mouse in the treatment of MCMV infection and myocarditis.
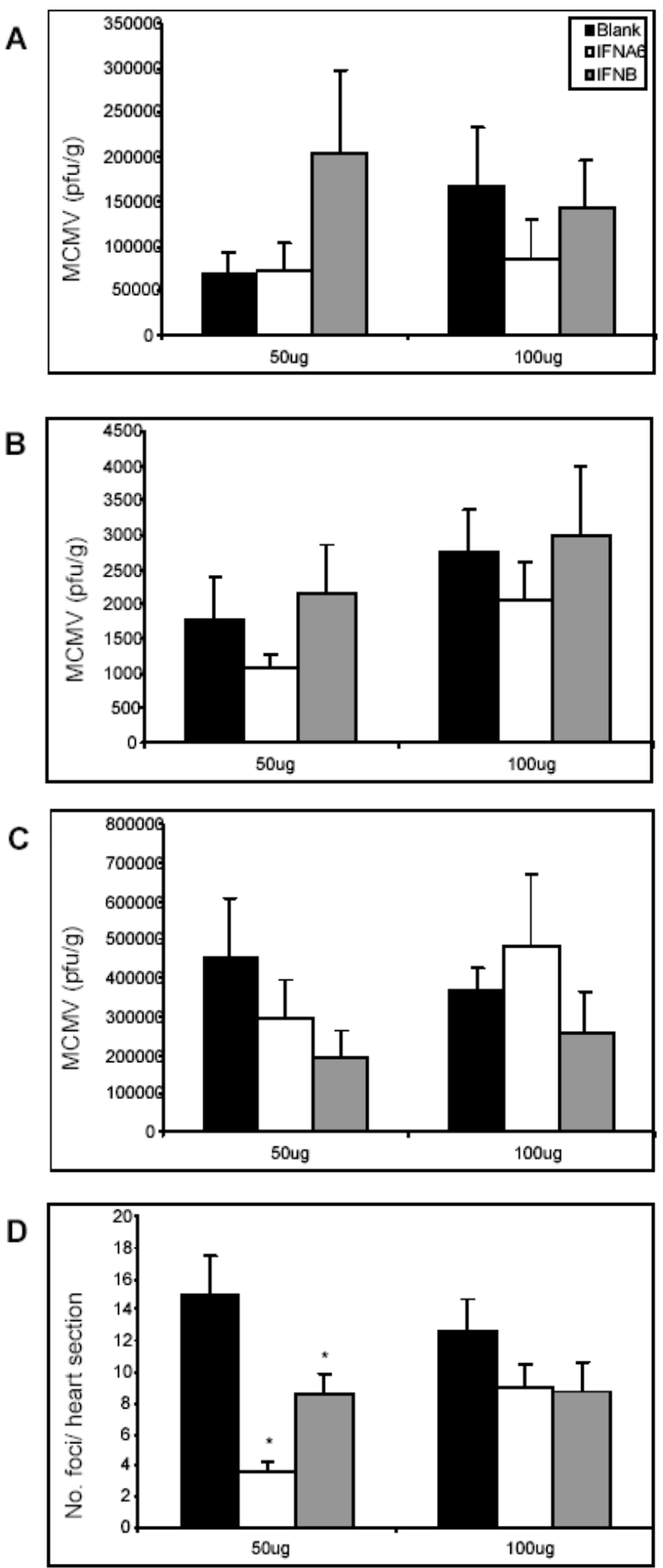

Fig. 1: The effect of DNA dosage in $I F N$ transgene treatment of MCMV infection and myocarditis. Bupivacaine-treated BALB/c mice were inoculated with doses of either $50 \mu \mathrm{g}$ or $100 \mu \mathrm{g}$ of Blank, IFNA6 or IFNB DNA at 14 days prior to viral challenge with $1 \times 10^{4}$ pfu of MCMV i.p. Viral titres were determined by plaque assay in (A) spleen and (B) liver at day 3 p.i. and (C) salivary glands at day 7 p.i. Results are expressed as mean pfu/g \pm SEM $(\mathrm{n}=5)$. (D) Myocarditis was evaluated at day 7 p.i. and is expressed as the number of foci per heart section \pm SEM $(n=5)$. *, Statistical significance $(\mathrm{p} \leq 0.05)$ compared with Blank treatment groups. 


\section{Assessment of DNA dose in co-administration of synergistic IFN subtypes}

The effect of combining two type I IFN subtypes was examined for determination of their possible synergistic efficacy in abrogating MCMV replication and disease. Previously we found synergistic properties of effective type I IFN subtypes when administered in combination (5). Combinations of Blank, IFNA6, IFNA9 and IFNB DNA were examined for their antiviral and antimyocarditic efficacy. In separate studies, BALB/c mice were inoculated i.m. with $200 \mu \mathrm{g}$ of each DNA construct (total $400 \mu \mathrm{g}$ DNA/mouse) or with $100 \mu \mathrm{g}$ of each DNA construct (total $200 \mu \mathrm{g}$ DNA/mouse) 14 days prior to challenge with $1 \times 10^{4}$ pfu MCMV by the i.p. route. Efficacious $I F N$ inoculation was demonstrated only at the lower dose of $200 \mu \mathrm{g}$ total DNA, as previously described (5), while the dosage of $400 \mu \mathrm{g}$ total DNA suppressed the efficacy associated with individual administration of these IFN subtypes.

In terms of antiviral efficacy, the $400 \mu \mathrm{g}$ IFNA6/Blank combination was not as effective as $200 \mu \mathrm{g}$ IFNA6 alone. In target tissues, IFNA6/Blank treatment showed no significant change with only a 1.8 -fold maximum reduction in virus titres (Fig, 2A, B, C) compared to IFNA6 alone treatment, resulting in 5.7-, 19- and 1.5-fold reductions in virus titres in the spleen, liver and salivary glands, respectively. The IFNA9 and IFNB treatments had no significant effects on viral titres with the exception of IFNB/Blank treatment at $400 \mu \mathrm{g}$ which significantly increased liver titres by 2.2 -fold at day 3 p.i. (Fig. 2B) as opposed to no significant change with $200 \mu \mathrm{g}$ IFNB alone at this timepoint. Overall, the $400 \mu \mathrm{g}$ combination of IFN/Blank did not demonstrate the level of antiviral protection observed with $200 \mu \mathrm{g}$ individual and combination IFN treatment, as previously described $(5,8)$.

In addition, we have previously described the synergistic properties of effective IFNs (IFNA6, $A 9$ and $B$ ) in combination to a total of $200 \mu \mathrm{g}$ DNA. Here, we also demonstrate that doses of $400 \mu \mathrm{g}$ DNA in combination is inferior for antiviral and antimyocarditic efficacy. Specifically, in the spleen, $200 \mu \mathrm{g}$ IFNA6/A9 reduced virus titres 3.0-fold and IFNA6/B reduced virus titres 50 -fold while $400 \mu \mathrm{g}$ of each of these IFN combination reduced virus titres by only 1.6 -fold and 1.3-fold, respectively. Similarly, in the liver, $200 \mu \mathrm{g} I F N A 6 / B$ reduced virus titres 3.2-fold and $I F N A 9 / B$ reduced virus titres 2.7 -fold while $400 \mu \mathrm{g}$ of these DNA vaccines marginally enhanced virus titres by 1.8 -fold and 1.5 -fold, respectively. IFN combinations had no detectable antiviral effects in the salivary glands.
A

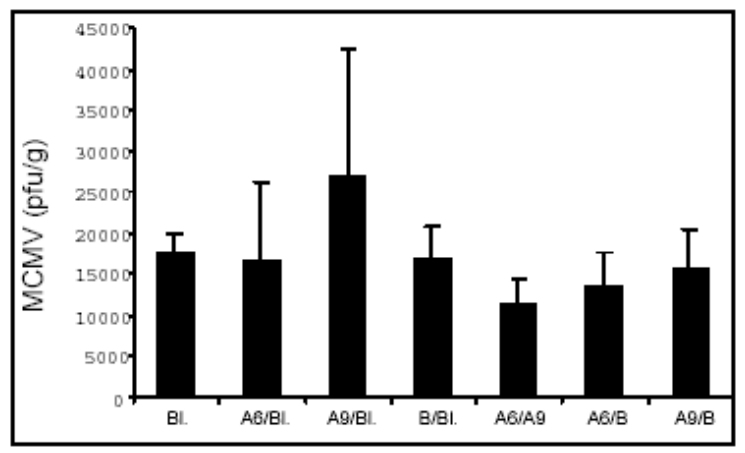

B

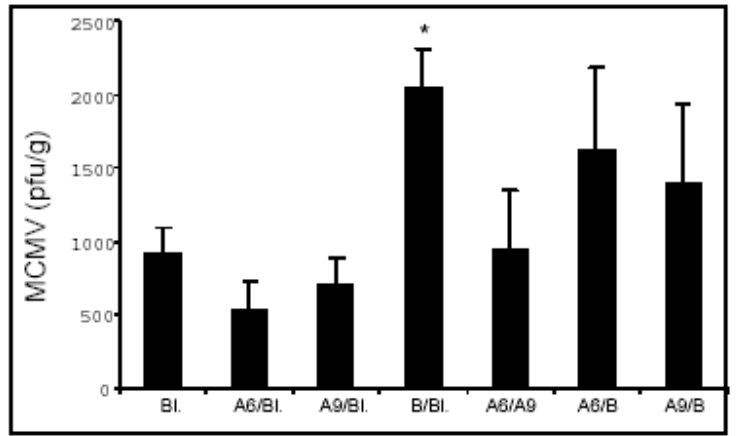

C

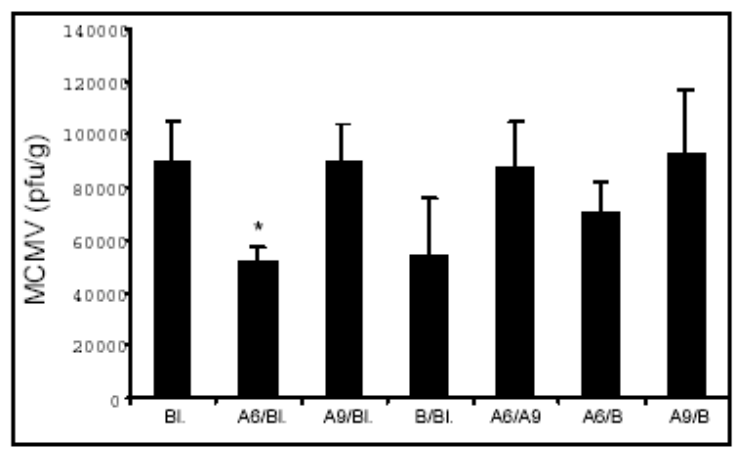

D

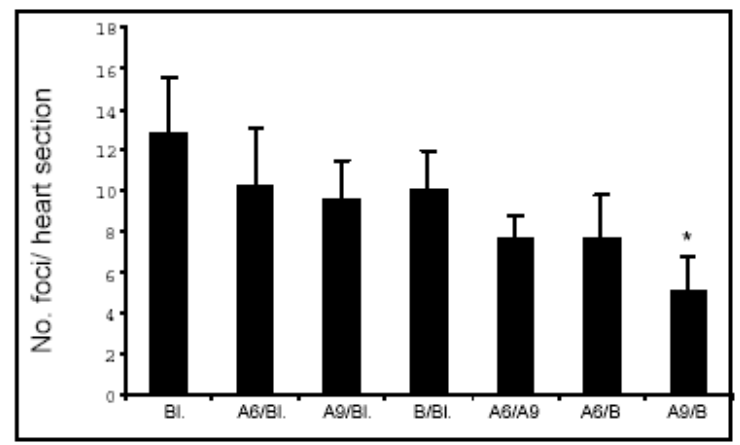

Fig. 2: Co-treatment with $400 \mu \mathrm{g} I F N$ subtype DNA does not affect acutephase MCMV infection or myocarditis. Bupivacaine treated BALB/c mice were inoculated with Blank vector or combinations of IFNA6/Blank, IFNA9/Blank, IFNB/Blank, IFNA6/A9, IFNA6/B, and IFNA9/B DNA at a dosage of $200 \mu \mathrm{g}$ per transgene to a total of $400 \mu \mathrm{g}$ DNA, bilaterally via the TA muscle. At 14 days post-inoculation, mice were challenged with $1 \times 10^{4} \mathrm{pfu}$ MCMV i.p. Viral titres were determined in (A) spleen and (B) liver at day 3 p.i. and (C) salivary glands at day 7 p.i. and are expressed as pfu/g tissue \pm SEM ( $n=5)$. (D) Myocarditis was evaluated at day 7 p.i. and is expressed as the number of foci per heart section $\pm \operatorname{SEM}(\mathrm{n}=5)$. *, Statistical significance $(\mathrm{p} \leq 0.05)$ compared with Blank treatment groups. Bl. denotes Blank expression vector. 
IFN treatment at $400 \mu \mathrm{g}$ DNA/mouse had no effect on acute phase MCMV-induced myocarditis with the exception of the $I F N A 9 / B$ treatment combination, which reduced disease severity by 2.5 -fold (Fig. 2D). However, this compared to a reduction in disease of 5.3-fold following administration of $200 \mu \mathrm{g} I F N A 9 / B$ and therefore is suboptimal. Notably, no significant reduction in acute myocarditis was observed with either IFNA6/Blank (1.2-fold), IFNA9/Blank (1.3-fold) or IFNB/Blank (1.3-fold, Fig. 2D) despite the protective effect previously observed following DNA treatment with these subtypes at doses of $200 \mu \mathrm{g} / \mathrm{mouse}$ with reductions of 4.7-, 2.0and 2.4-fold, respectively (8). Thus co-inoculation of effective type I IFNs with Blank vector at a total of $400 \mu \mathrm{g} / \mathrm{mouse}$ resulted in abrogation of efficacy previously observed in this animal model.

\section{Pre-medication with bupivacaine is critical for IFNA6 immunotherapy}

We have previously demonstrated the superior efficacy of IFNA6 inoculation, which has potent antiviral effects and reduces disease pathogenesis in the MCMV model (8). Our standard inoculation protocol includes the treatment of mice 5 days prior to DNA inoculation with $20 \mu \mathrm{L} 0.5 \%$ bupivacaine given bilaterally to the TA muscles. Here, we investigated the necessity of bupivacaine treatment prior to IFNA6 administration. Mice were either treated or not treated with bupivacaine 5 days prior to IFNA6 DNA inoculation and subsequently challenged with $1 \times 10^{4}$ pfu MCMV. The effect of bupivacaine treatment on the antiviral response and myocarditis was evaluated.

Treatment with the IFNA6 transgene with bupivacaine demonstrated reduced MCMV titres in the spleen and liver at day 3 p.i. (Fig. 3A and B) and the salivary gland at day 7 p.i. (Fig. 3C). However, without the administration of bupivacaine, a significant reduction in MCMV was observed only in the liver at day 3 p.i. (Fig. 3B) with no effect observed in other tissues. Similarly, IFNA6 inoculation significantly reduced acute-phase myocarditis whilst IFNA6 administration without bupivacaine had no effect on disease severity in the myocardium (Fig. 3D). Therefore, maximal efficacy for MCMV infection was achieved with bupivacaine premedication i.m.

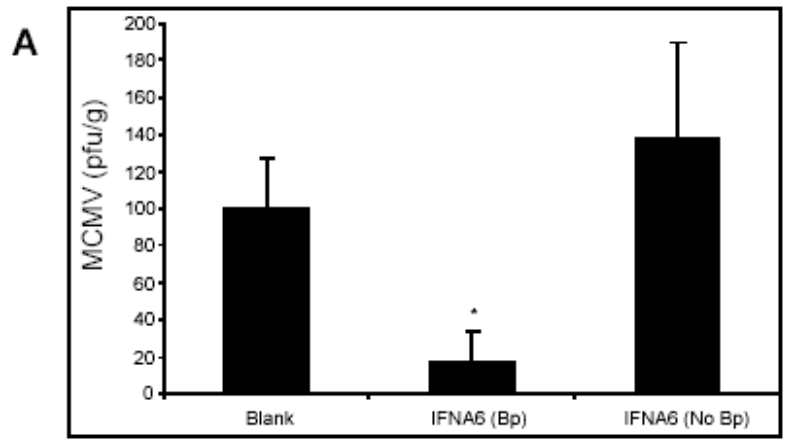

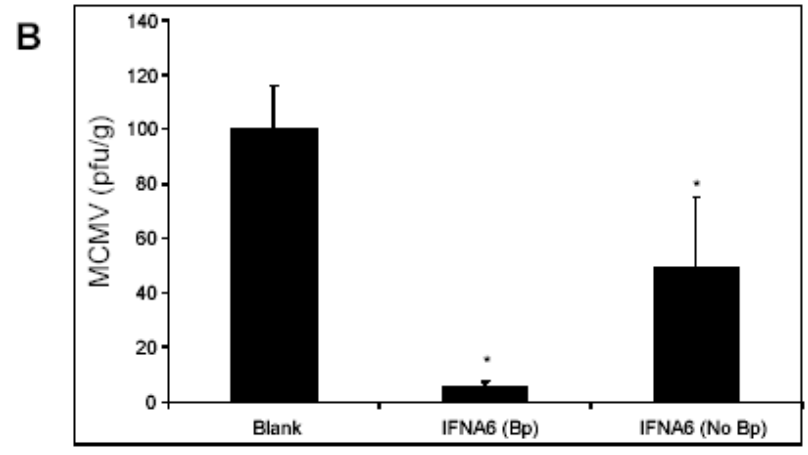
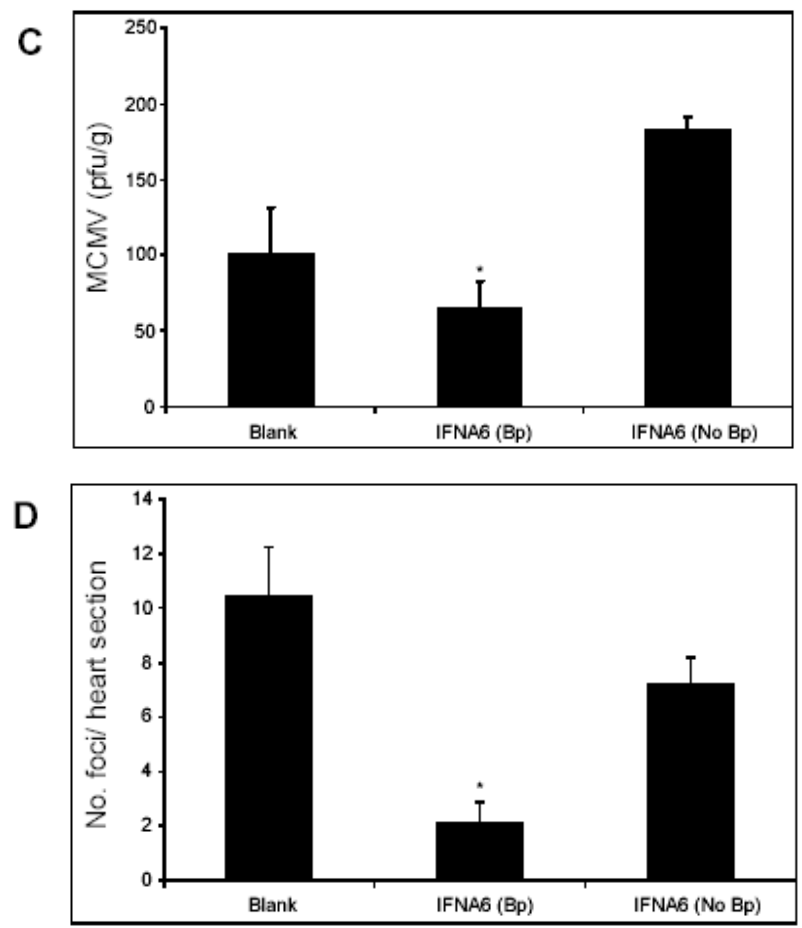

Fig. 3: $I F N$ DNA inoculation without bupivacaine treatment is ineffective against MCMV infection and myocarditis. BALB/c mice were either not treated (No $\mathrm{Bp}$ ) or treated $(\mathrm{Bp})$ with bupivacaine 5 days prior to DNA inoculation with $200 \mu \mathrm{g}$ of either Blank or IFNA6 vector DNA and subsequently challenged with $1 \times 10^{4}$ pfu of MCMV i.p 14 days later. Viral titres were determined by plaque assay in (A) spleen and (B) liver at day 3 p.i., and (C) salivary glands at day 7 p.i. Results expressed as \% Blank treatment group $\pm \operatorname{SEM}(n=5)$. (D) Myocarditis was evaluated at day 7 p.i. and is expressed as the number of foci per heart section $\pm \operatorname{SEM}(n=5) .{ }^{*}$, Statistical significance $(\mathrm{p} \leq 0.05)$ compared with Blank treatment groups.

\section{Combinational IFN immunotherapy for $C M V \mathrm{gB}$ DNA vaccination}

Previous studies have revealed the capacity of specific type I IFN DNAs, when co-immunised with $g B$ DNA, to enhance the protection afforded by the $g B$ DNA vaccine against MCMV replication (9). Here we examine the capacity of $g B / I F N A 6 / I F N B$ to enhance the protection previously observed against MCMV infection afforded by $g B$ DNA vaccines (9), DNA encoding specific IFN subtypes (8), and combinations of IFN subtypes (5). Each mouse was infected with $1 \times 10^{4} \mathrm{PFU}$ of MCMV 14 days post-vaccination with either Blank $(200 \mu \mathrm{g})$, 
$g B(200 \mu \mathrm{g})$ or combination $g B / I F N A 6 / I F N B(50 \mu \mathrm{g}, 50 \mu \mathrm{g}$, $100 \mu \mathrm{g}$ respectively) DNA constructs. The addition of the $I F N$ combination as adjuvant to the $g B$ vaccine did not alter the vaccines antiviral efficacy in the spleen (Fig. 4A). However, inoculation of IFNA6/IFNB DNA with $g B$ led to a significant reduction in MCMV replication in the liver at day 7 p.i. (Fig. 4B). In addition, the combined $g B / I F N A 6 / I F N B$ DNA vaccine significantly decreased both acute (Fig. 4C) and chronic (Fig. 4D) myocarditis in comparison to the Blank and $g B$ DNA vaccinated groups.

\section{Discussion}

Here we describe a detailed study focusing on optimization of the inoculation protocol for type I IFN subtype DNA constructs in the MCMV model. Delivery of either individual or combination IFN subtypes allows for investigation of the biological function and synergy of $I F N$ in vivo. In addition, the delivery of IFN DNA subtypes can be examined for enhancement of protective immunity afforded by viral vaccination of mice.

Contributing factors for effective DNA treatment examined in this study included optimal dose determination for IFN DNA inoculation. Previous studies in our laboratory using mammalian expression plasmid administered IFN $\alpha$ have effectively used inoculations of $200 \mu \mathrm{g}$ DNA (5-8). Data presented in this study demonstrates that lower doses of $50 \mu \mathrm{g}$ and $100 \mu \mathrm{g}$ type I IFN DNA, inoculated 14 days prior to virus, were ineffective in reducing viral replication. Interestingly, $50 \mu \mathrm{g}$ DNA effectively reduced acute myocarditis while $100 \mu \mathrm{g}$ IFN had no effect. Co-inoculation of effective type I IFNs (total $400 \mu \mathrm{g} /$ mouse) resulted in abrogation of the antiviral efficacy and suppression of disease previously observed in this model $(5,8)$. It may be that excess doses of IFN DNA $(400 \mu \mathrm{g})$ render the IFN DNA treatment ineffective at reducing antiviral titres and myocarditis and altering cytokine profiles. The exception to this was treatment with $I F N A 9 / B$, which reduced disease (2.5-fold) and circulating IFN $\gamma$ titres (data not shown), however, this was not as effective as the $200 \mu \mathrm{g}$ combination IFNA9/B which reduced myocarditis by 5.3-fold (5). Therefore, dosage of DNA was found to be crucial for therapeutic outcome. In this study, we have demonstrated that both insufficient and excess DNA suppressed the efficacy of type I IFN DNA treatment. Dose-response curves from previous studies demonstrate optimal DNA doses at 100-200 $\mu \mathrm{g}$ with decline in efficacy at higher concentrations (14). Effective gene immunotherapy has previously been demonstrated with doses of $200 \mu \mathrm{g} /$ mouse for IFN $\gamma \mathrm{R}-\mathrm{Fc}$ (15) and IL-4/IgG1 (16) and type I IFNs (7). Similarly, we have shown that type I IFN DNA inoculation is most effective at $200 \mu \mathrm{g} / \mathrm{mouse}$, administered both as individual subtypes and as combination treatments. This indicates that effective type I IFN DNA inoculation is dose-dependent which is consistent with findings in other models of DNA vaccination $(1,13)$.
A

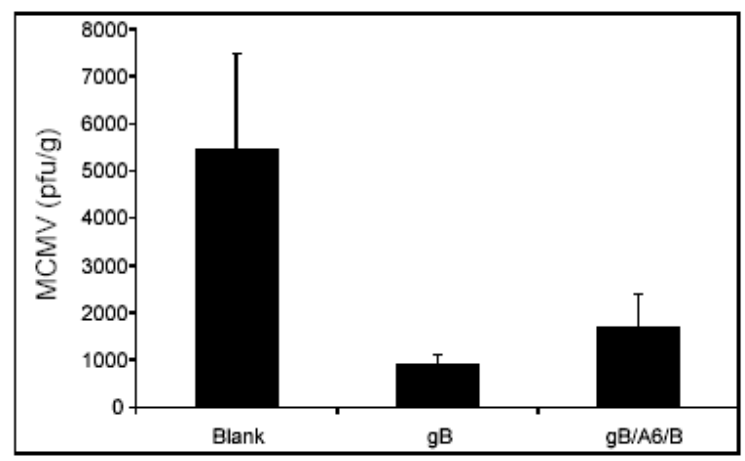

B

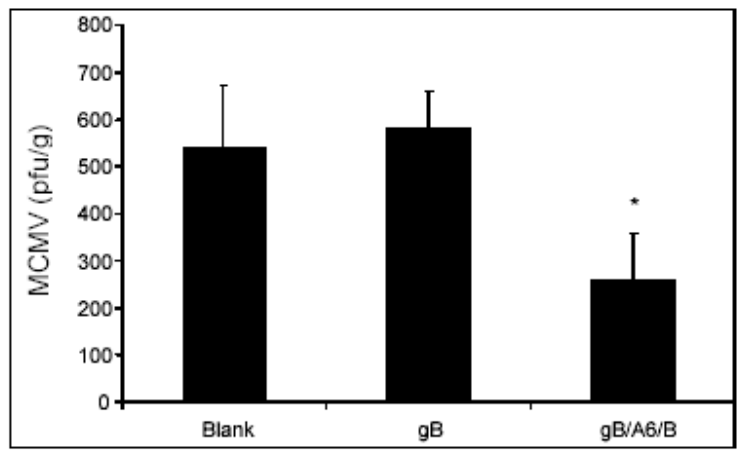

C

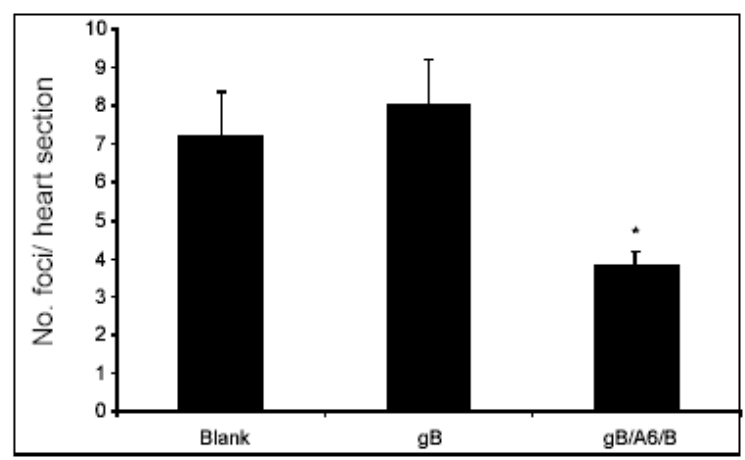

D

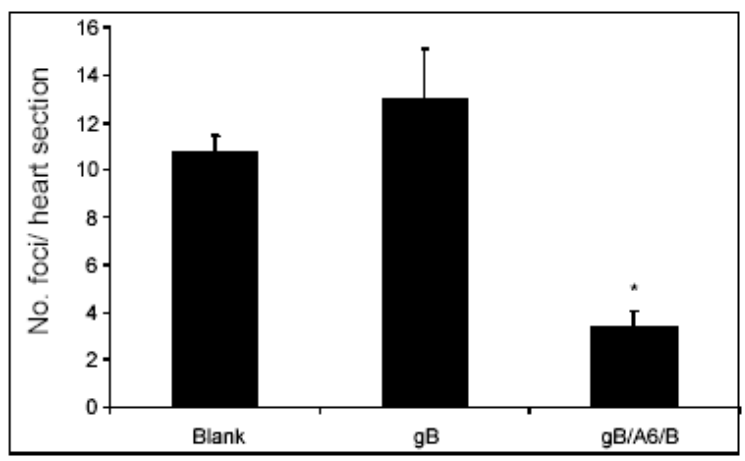

Fig. 4: Combinational $I F N$ DNA co-administration with gB improves vaccine efficacy in vivo. BALB/c mice were vaccinated with either Blank, $g B$, or gB/IFNA6/IFNB DNA ( $200 \mu \mathrm{g} /$ mouse) 14 days prior to i.p. inoculation with $1 \times 10^{4} \mathrm{pfu}$ of MCMV. Viral titres were determined by plaque assay in (A) spleen at day 3 p.i. and (B) liver at day 7 p.i. Myocarditis was evaluated at (C) day 10 p.i. and (D) day 56 p.i. Myocarditis is expressed as the number of foci per heart section \pm SEM $(\mathrm{n}=5)$. *, Statistical significance $(\mathrm{p} \leq 0.05)$ compared with $g B$ DNA vaccinated group. 
It is widely believed that inoculation of DNA constructs into regenerating muscle contributes to enhanced uptake and expression of transgenes $(12,17)$. Bupivacaine treatment is an efficient inducer of myogenesis prior to plasmid inoculation (12), however, evidence for and against this treatment is conflicting. In this study, plasmid administration without bupivacaine treatment was generally ineffective for all aspects evaluated with the exception that IFNA6 reduced viral titres in the liver. Despite this, viral titres in the liver were 9.4-fold greater in the group not treated with bupivacaine compared to the bupivacaine treated group. Similarly, viral titres following IFNA6 inoculation without bupivacaine treatment increased 7.8-fold and 2.8-fold in the spleen and salivary gland respectively compared to the bupivacaine treated group. Furthermore, myocarditis increased 3.5-fold without bupivacaine treatment compared with bupivacaine treatment following IFNA6 treatment. Therefore, we demonstrated that bupivacaine treatment is a necessary component of the optimal inoculation program in DNA inoculation in the MCMV model.

We previously investigated the timing of DNA administration and demonstrated that optimal efficacy was achieved when mice were treated with IFN DNA 14 days prior to viral challenge (5). Treatment with IFNA6 and IFNB 2 days before and 2 days after viral infection proved ineffective during the acute phase of viral infection and disease. Interestingly, IFN DNA treatment 2 days prior to viral infection reduced the chronic phase of myocarditis. The limited efficacy of IFN treatment at these time-points, however, is largely expected given that systemic IFN expression peaks at day 28 following IFN treatment and is not significantly increased in comparison to Blank groups until day 14 (5). In addition, endogenous IFN, produced as part of the immune response to viral invasion, peaks 2 days following LCMV infection and may therefore mask IFN transgene expression at this time (18). We postulate that type I IFN administrated at later time-points is insufficiently expressed prior to endogenous cytokine production in response to virus. Surprisingly, IFNB treatment 2 days prior to virus contributed to a pathogenic immune response. Indeed, another study has demonstrated a distinct role for IFN $\beta$ as an "immediate-early response gene" following viral infection. This group suggests that IFN $\beta$ must be synthesized for induction of a delayed IFN gene set (including $\alpha 2, \alpha 5, \alpha 6$ and $\alpha 8$ ) (19). Potentiation of IFN $\alpha$ cytokines by IFN $\beta$ may exacerbate the immune response and lead to detrimental responses in this model.

Finally, the optimization of the IFN DNA inoculation protocol was trialed as adjuvant immunotherapy for a CMV-specific DNA vaccine. Clearly, this study confirms the superiority of the combined viral $g B / I F N A 6 / I F N B$ DNA vaccine to that of the viral $g B$ DNA vaccine in providing protection from MCMV infection and myocarditis. Strikingly, the synergistic effects of the $g B / I F N A 6 / I F N B$ DNA vaccine were found to significantly decrease inflammation within the myocardium during both the acute and chronic phases to levels previously seen in MCMV- infected C57BL/6 mice which are resistant to the development of post-viral myocarditis (20). This research into the synergistic effects of the $g B / I F N A 6 / I F N B$ DNA vaccine has revealed the potential of specific IFNs and immunogenic proteins to act as an enhanced immunotherapy when combined and administered as a single DNA vaccine.

Our observations using $200 \mu \mathrm{g}$ DNA/mouse show that treatment with $g B / I F N A 6 / I F N B$ DNA was ineffectual in comparison to $I F N A 6 / I F N B(5)$ and $g B / I F N B(9)$, at decreasing MCMV replication in target organs. However, results from these previous studies revealed no correlation between virus load and subsequent development of acute myocarditis. Coimmunisation of IFNA6/IFNB reduced viral titres in both spleen and liver, with no improvement in acute phase myocarditis, but reduced myocarditis in the chronic phase (5). Whereas, co-immunisation with $g B / I F N B$ caused significantly reduced MCMV titres in target organs as well as reduced acute and chronic phase myocarditis (9). Therefore results from this study and previous experiments follow the speculation of viral load in target organs not affecting acute phase myocarditis, but possibly being a factor in the latter stage development of autoimmune myocarditis. Furthermore, the $g B / I F N A 6 / I F N B$ vaccine was found to be of similar efficacy to the $g B / I F N B$ vaccine in the general suppression of MCMV-induced myocarditis. Thus viral $g B / I F N B$ vaccination using our optimised delivery of DNA constructs would be the preferred treatment of choice.

Therapeutic application of combined IFN subtypes to DNA vaccines may provide an extensive array of new possibilities for future research into protection from diseases that were otherwise thought incurable or exhaustive of any further applications. Clinical trials into DNA viral vaccines for HIV and influenza virus have arisen due to the urgent need for a protective vaccine for these infectious diseases. Trials have used DNA vaccines encoding viral proteins such as influenzaspecific hemagglutinin (21), and HIV-specific gp120 and gp160 (22), shown only to provide partial protection. However, with the knowledge gained on type I IFNs and their synergistic properties from our studies, the application of combined IFNs could possibly be used in improving the efficacy of these DNA vaccines.

In summary, we describe in this study the optimization of intramuscular expression of DNA transgenes encoding IFN $\alpha / \beta$ subtypes for improved protection of mice from MCMVinduced myocarditis. We have successfully utilised the DNA delivery method to examine the therapeutic antiviral and antimyocarditic effects of both individual and combinational IFN subtypes in vivo. This experimental system can also be applied in DNA vaccine trials to study the therapeutic efficacy of type I IFN subtypes in host protection from other virus infections. 


\section{ACKNOWLEDGMENTS}

This work is supported by the National Health and Medical Research Council of Australia (No. 990393) with funding from the Western Australian Biomedical Research Institute, Murdoch University. The authors thank Vical Inc. (CA) for the generous donation of the plasmid vector, pkCMVint.

\section{REFERENCES}

1. Gherardi MM, Ramirez JC, Esteban M. Interleukin-12 (IL-12) enhancement of the cellular immune response against human immunodeficiency virus type 1 Env antigen in a DNA prime/ vaccinia virus boost vaccine regimen is time and dose dependent: suppressive effects of IL-12 boost are mediated by nitric oxide. $J$ Virol 2000; 74:62786286.

2. Noormohammadi AH, Hochrein H, Curtis JM, Baldwin TM, Handman E. Paradoxical effects of IL-12 in leishmaniasis in the presence and absence of vaccinating antigen. Vaccine 2001; 19:4043-4052.

3. Prud'homme GJ, Lawson BR, Chang Y, Theofilopoulos AN. Immunotherapeutic gene transfer into muscle. Trends Immunol 2001; 22:149-155.

4. Min W, Lillehoj HA, Burnside J, Weining KC, Staeheli P, Zhu JJ. Adjuvant effects of $I L-1 \beta, I L-2, I L-8, I L-15, I F N \alpha$, $I F N \gamma, T G F \beta 4$ and lymphotactin on DNA vaccination against Eimeria acervulina. Vaccine 2002; 20:267-274.

5. Bartlett EJ, Cull VS, Brekalo NL, Lenzo JC, James CM. Synergy of type I interferon-A6 and interferon-B naked DNA immunotherapy for cytomegalovirus infections. Immunol Cell Biol 2002; 80:425-435.

6. Lawson CM, Yeow WS, Lee CM, Beilharz MW. In vivo expression of an interferon- $\alpha$ gene by intramuscular injection of naked DNA. J Interferon Cytokine Res 1997; 17:255-261.

7. Yeow WS, Lawson CM, Beilharz MW. Antiviral activities of interferon- $\alpha$ subtypes in vivo. J Immunol 1998; 160:2932-2939.

8. Cull VS, Bartlett EJ, James CM. Type I interferon gene therapy protects against cytomegalovirus-induced myocarditis. Immunol 2002; 106:428-437.

9. Cull VS, Broomfield S, Bartlett EJ, Brekalo NL, James CM. Coimmunisation with type I IFN genes enhances protective immunity against cytomegalovirus and myocarditis in gB DNA-vaccinated mice. Gene Therapy 2002; 9:1369-1378.

10. Horton HM, Anderson D, Hernandez P, Barnhart KM, Norman JA, Parker SE. A gene therapy for cancer using intramuscular injection of plasmid DNA encoding interferon $\alpha$. Proc Nat Acad Sci USA 1999; 96:1553-1558.

11. Noisakran S, Campbell IL, Carr DJJ. Ectopic expression of DNA encoding IFN- $\alpha 1$ in the cornea protects mice from herpes simplex virus type 1-induced encephalitis. $J$ Immunol 1999;162:4184-4190.
12. Danko I, Wolff JA. Direct Gene Transfer into Muscle. Vaccine 1994; 12:1499-1502.

13. Kusakabe K, Xin K, Katoh H, Sumino K, Hagiwara E, Kawamoto S, Okuda K, Miyagi Y, Aoki I, Nishioka K, Klinman D, Okuda K. The timing of GM-CSF expression plasmid administration influences the Th1/ Th2 response induced by an HIV-1-specific DNA vaccine. $J$ Immunol 2000; 164:3102-3111.

14. Moelling K. DNA for genetic vaccination and therapy. Cytokines, Cell Molec Therapy 1997; 3:127-136.

15. Prud'homme GJ, Chang Y. Prevention of autoimmune diabetes by intramuscular gene therapy with a nonviral vector encoding an interferon- $\gamma$ receptor/ IgG1 fusion protein. Gene Therapy 1999; 6:771-777.

16. Chang Y, Prud'homme GJ. Intramuscular administration of expression plasmids encoding interferon- $\gamma$-receptor/ IgG1 or IL-4/ IgG1 chimeric proteins protects from autoimmunity. J Gene Med 1999; 1:415-423.

17. Vitadello M, Schiaffino MV, Picard A, Scarpa M, Schiaffino S. Gene transfer in regenerating muscle. Human Gene Therapy 1994; 5:11-18.

18. Biron CA. Role of early cytokines, including alpha and beta interferons (IFN- $\alpha / \beta)$, in innate and adaptive immune responses to viral infections. Sem Immunol 1998;10:383390.

19. Marié I, Durbin JE, Levy DE. Differential viral induction of distinct interferon- $\alpha$ genes by positive feedback through interferon regulatory factor-7. EMBO $J$ 1998; 17:66606669.

20. Lenzo JC, Fairweather D, Cull V, Shellam GR, James (Lawson) CM. Characterisation of murine cytomegalovirus myocarditis: cellular infiltration of the heart and virus persistence. $J$ Mol Cell Cardiol 2002; 34:629-640.

21. Ulmer JB. Influenza DNA vaccines. Vaccine 2002; 20:S74-76.

22. MacGregor RR, Ginsberg R, Ugen KE, Baine Y, Kang $\mathrm{CU}$, Tu XM, Higgins T, Weiner DB, Boyer JD. T-cell responses induced in normal volunteers immunized with a DNA-based vaccine containing HIV-1 env and rev. AIDS 2002; 16:2137-2143. 


\section{PROTOCOLS}

\section{Protocol 1: Large-scale preparation of plasmid DNA}

\section{Reagents:}

Terrific broth (Solution A: 1.3\% Tryptone Peptone (w/v) [Difco \#211705]; 2.67\% Yeast extract [Becton Dickinson \#211929]; 0.44\% Glycerol (v/v) [Univar \#242-500ML]; Solution B: 719mM K ${ }_{2} \mathrm{HPO}_{4}\left[\mathrm{BDH}\right.$ AnalaR \#104363A]; $169 \mathrm{mM} \mathrm{KH}_{2} \mathrm{PO}_{4}\left[\mathrm{Univar}^{2}\right.$ \#391-500G]). Autoclave solution A and B separately. Combine the two solutions at a ratio of 9:1 respectively.

TEG swelling buffer (25mM Tris [Invitrogen \#15504-020], pH8.0; 10mM EDTA [BDH AnalaR 10093.5V]; 0.9\% Glucose (w/v) [Univar \#783-500G]). Store at $4^{\circ} \mathrm{C}$.

Lysis Buffer (0.2M NaOH [Univar \#482-500G]; 1\% SDS [BDH GPR \#301754L]). Prepare just prior to use.

High Salt Neutralisation buffer (2.5M Potassium acetate [BDH \#295814P]; 5\% Formic acid (v/v) [Univar \#2471-500]).

$\mathrm{LiCl}$ solution (5M LiCl [BDH GPR \#290674B]; 1\% MOPS (w/v) [Sigma \#M-9024], pH to 8.0). Autoclave and store at $4^{\circ} \mathrm{C}$.

Tris-buffered phenol. Melt phenol [Sigma \# P-1037] at $65^{\circ} \mathrm{C}$ and extract with $1 \mathrm{M}$ Tris, $\mathrm{pH} 8.0$ by stirring O/N. Change buffer 3 times over a $24 \mathrm{~h}$ period or until $\mathrm{pH}$ of the aqueous phase is $>7.6$. Extract once more with $0.1 \mathrm{M}$ Tris, $\mathrm{pH} 8.0$ and store under equilibrium buffer @ $44^{\circ} \mathrm{C}$ in the dark.

Phenol/ chloroform/isoamyl alcohol (25:24:1). Add an equal volume of chloroform [Sigma \#C-2432]/isoamyl alcohol [Sigma \#1$3643](24: 1 \mathrm{v} / \mathrm{v})$ to Tris-buffered phenol and store under $0.1 \mathrm{M}$ Tris, $\mathrm{pH} 8.0$ at $4^{\circ} \mathrm{C}$ in the dark.

Isopropanol [Univar \#425-2.5]

Ethanol [Sigma \#E-7023]

RNase, DNase-free [Roche \#1119915]

Sodium acetate [Univar \#679-500G]

\section{Protocol:}

1. Prepare bacterial cultures containing the required plasmid by inoculating a loopful of glycerol stock plasmid into 1L Terrific Broth (containing appropriate antibiotic). Incubate $\mathrm{O} / \mathrm{N}$ at $37^{\circ} \mathrm{C}$ with shaking.

2. Centrifuge at $2,500 \mathrm{~g}, 4^{\circ} \mathrm{C}, 10 \mathrm{~min}$.

3. Resuspend pellet in $50 \mathrm{~mL}$ TEG swelling buffer at $4^{\circ} \mathrm{C}$. Add $100 \mathrm{~mL}$ Lysis buffer and incubate on ice for $10 \mathrm{~min}$.

4. Add $75 \mathrm{~mL}$ High Salt Neutralisation buffer (a white precipitate will form). Centrifuge at $2,500 \mathrm{~g}, 4^{\circ} \mathrm{C}, 10 \mathrm{~min}$.

5. Pour the supernatant through sterile gauze and add an equal volume of isopropanol. Centrifuge at $2,500 \mathrm{~g}, 4^{\circ} \mathrm{C}, 10 \mathrm{~min}$.

6. Pour off the supernatant and resuspend the pellet in $20 \mathrm{~mL}$ double-deionised $\mathrm{H}_{2} \mathrm{O}\left(\mathrm{dd}_{2} \mathrm{O}\right)$. Transfer the solution to a polypropylene tube.

7. Add $20 \mathrm{~mL} \mathrm{LiCl}$ solution at $4^{\circ} \mathrm{C}$, mix and stand on ice for $10 \mathrm{~min}$. Centrifuge at $2,500 \mathrm{~g}, 4^{\circ} \mathrm{C}, 15 \mathrm{~min}$. Transfer the supernatant to a fresh polypropylene tube and discard pellet.

8. Extract the supernatant with an equal volume of phenol/ chloroform/ isoamyl alcohol. Centrifuge at $1,800 \mathrm{~g}, 20^{\circ} \mathrm{C}, 5 \mathrm{~min}$.

9. Transfer the aqueous phase to a polypropylene tube and add $40 \mathrm{~mL}$ isopropanol. Mix by inversion. Centrifuge at $2,500 \mathrm{~g}, 4^{\circ} \mathrm{C}$, $10 \mathrm{~min}$. Discard the supernatant.

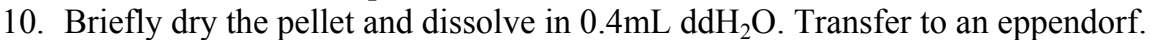

11. Add $4 \mu \mathrm{L}$ RNase and incubate at $37^{\circ} \mathrm{C}$ for $15 \mathrm{~min}$.

12. Add $40 \mu \mathrm{L} 3 \mathrm{M}$ sodium acetate $(\mathrm{pH} 4.8)$ and $1 \mathrm{~mL}$ ethanol. Leave on dry ice for $15 \mathrm{~min}$ (or at $-20^{\circ} \mathrm{C}$ overnight). Centrifuge at $13,000 \mathrm{~g}, 30 \mathrm{~min}$ at $20^{\circ} \mathrm{C}$.

13. Wash pellet $1 \mathrm{~mL} 70 \%$ ethanol and centrifuge at $13,000 \mathrm{~g}, 5 \mathrm{~min}$ at $20^{\circ} \mathrm{C}$.

14. Briefly dry pellet and resuspend in $\mathrm{dd}_{2} \mathrm{O}$.

15. Read OD@260nm and 280nm.

\section{Protocol 2: Intramuscular immunisation of DNA constructs}

\section{Reagents:}

Insulin syringes, Ultra fine needle 29 Gauge $(0.33 \mathrm{~mm} \times 12.7 \mathrm{~mm})$ [Becton Dickinson \#326769]

Marcain, 0.5\% Bupivacaine [Astra \#6641000]

Pyrogen-free saline, 0.9\% sodium chloride [Astra Zeneca \#217904] 


\section{Protocol:}

1. Mice (4 week old male BALB/c) are restrained by grasping the skin behind the ears with the thumb and index finger and tucking the tail underneath the little finger (as per i.p. injections). The legs are then sprayed with $70 \%$ ethanol to enable clear identification of the tibialis anterior (TA) muscle for intramuscular inoculation.

2. $20 \mu \mathrm{L} 0.5 \%$ bupivacaine inoculated bilaterally via TA muscle.

3. 5 days later, DNA constructs $(200 \mu \mathrm{g} /$ mouse) are inoculated by the intramuscular route. Dilute DNA in $50 \mu \mathrm{L}$ volume of sterile pyrogen-free saline $(4 \mu \mathrm{g} / \mu \mathrm{L})$.

4. Inoculate mice with $25 \mu \mathrm{L}$ DNA preparation bilaterally via the TA muscle.

5. Allow 14 days for intramuscular protein expression from the DNA constructs prior to viral challenge. 